\title{
Risk and protection in the gut
} ce

\section{the complex \\ interplay \\ between \\ intestinal \\ homeostasis, dysbiosis and disease}

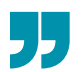

Maintenance of the intestinal ecosystem involves a complex interplay between the host, resident microbial communities and invading pathogens. The host-associated microbiota has the capacity to prevent the establishment of new species, especially pathogens (colonization resistance), and the intestinal mucosal surface functions as a defensive barrier against pathogenic microorganisms. Defects in intestinal epithelial barrier function have been linked to an altered intestinal microenvironment, dysbiosis, pathogen expansion and mucosal inflammation, and those shifts have been implied in the pathogenesis of inflammatory bowel disease (IBD). However, the link between the resident microbiota and colitis is not well understood. Two studies now describe how a commensal can either protect against or promote colitis, and a third study shows how colitis can affect the disease severity of a subsequent infection.

Herp, Brugiroux et al. hypothesized that mutant mice that lack the inner mucus layer and exhibit

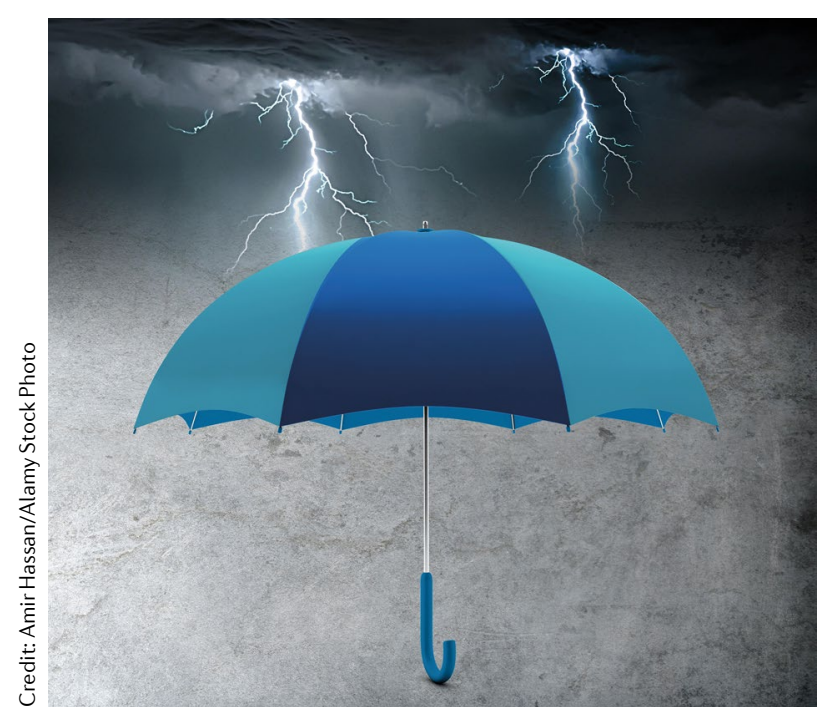

mucosal barrier defects are highly susceptible to enteric infection with non-typhoidal Salmonella enterica subsp. enterica serovar Typhimurium and S. Typhimurium-induced colitis in the streptomycin mouse model. However, in contrast to mucus-proficient littermates, mutant animals exhibited delayed onset of colitis and reduced tissue invasion. The protective effect in mutant mice correlated with alterations in the composition of the gut microbiota. Specifically, comparative microbiome profiling revealed that Mucispirillum schaedleri, a member of the phylum Deferribacteres present in the intestinal microbiota of mice and humans, was enriched in the caecum of antibiotic-treated mutant mice compared with the wild type. Gnotobiotic mice colonized with M. schaedleri showed significantly reduced gut inflammation compared with control animals following infections with $S$. Typhimurium, whereas pathogen loads in the gut were unchanged. This suggests that M. schaedleri protects the animals from $S$. Typhimurium-induced colitis but not from gut luminal colonization. Transcriptome analysis showed that several genes that are essential for growth, metabolism and the function of the type III secretion system were downregulated in $S$. Typhimurium in the presence of M. schaedleri, which leads to a decrease in virulence, tissue invasion and inflammatory responses. The authors conclude that $M$. schaedleri confers protection against $S$. Typhimurium-induced colitis in mice.

In a second study, Caruso et al. used mice harbouring mutations in previously identified IBDsusceptibility genes. Specifically, mice deficient in NOD2 and phagocyte NADPH oxidase were fostered by dams colonized with different microbiota. Interestingly, the authors found that only one group of mutant mice harbouring a distinct microbiota developed spontaneous colitis, suggesting that a specific bacterial species within this microbial community may trigger disease. Although the overall gut microbial composition did not differ between wild-type animals and double-mutant mice fostered by the same dams, the authors noticed a marked accumulation of M. schaedleri in the mutants that developed colitis. They suggest that decreased neutrophil recruitment and NADPH oxidase activity in the mutant mice promoted the accumulation and intestinal invasion of $M$. schaedleri and that outgrowth of the intestinal bacterium can induce disease in immunocompromised hosts.

In an unrelated third study, Saleh et al. investigated the mechanism underlying the observation that Clostridium difficile infection (CDI) is more common and severe in patients with IBD. The authors report that prior colitis induces a population of T helper 17 cells in mice that persists following recovery from gut inflammation. Specifically, they showed that aberrant immune responses caused by the increased levels of the cytokine produced by these cells, interleukin-17A, was associated with a worse disease outcome following CDI.

In summary, the studies highlight the complex interplay between intestinal homeostasis, dysbiosis and disease.

Andrea Du Toit

ORIGINAL ARTICLES Herp, S. \& Brugiroux, S. et al. Mucispirillum schaedleri antagonizes Salmonella virulence to protect mice against colitis. Cell Host Microbe https://doi.org/10.1016/j.chom. 2019.03 .004 (2019) | Caruso, R. A specific genemicrobe interaction drives the development of Crohn's disease-like colitis in mice. Sci. Immunol. 4, eaaw4341 (2019) | Saleh, M. M. et al. Colitisinduced Th17 cells increase the risk for severe subsequent Clostridium difficile infection. Cell Host Microbe https://doi.org/10.1016/j.chom. 2019.03.003 (2019) 